\title{
Composite films combining electrospun fiber network and epitaxial oxide by chemical solution deposition
}

Albert Calleja ${ }^{1,2}$, Jordi Sort ${ }^{3}$, Susagna Ricart ${ }^{1}$, Xavier Granados ${ }^{1}$, Xavier Palmer ${ }^{1}$, Valentina Roxana Vlad ${ }^{2}$, Teresa Puig ${ }^{1}$, Xavier Obradors ${ }^{1}$

${ }^{1}$ Institut de Ciència de Materials de Barcelona (ICMAB-CSIC,) Campus Universitat Autònoma de Barcelona, 08193 Bellaterra, Spain

${ }^{2}$ Oxolutia SL, Edifici Eureka, Parc de Recerca UAB, Campus de la Universitat Autònoma de Barcelona, 08193 Bellaterra, Spain

${ }^{3}$ Institució Catalana de Recerca i Estudis Avançats (ICREA) and Departament de Física, Facultat de Ciències, Edifici Cc, Universitat Autònoma de Barcelona, 08193, Bellaterra, Spain

Corresponding author: Albert Calleja, acalleja@icmab.es , Tel. +34 9358018 53, Fax. $+34935805729$ 


\section{$\underline{\text { Abstract }}$}

We report the preparation of a novel type of composites films by chemical solution deposition. It consists of an epitaxial oxide on a single crystal template inside which an oxide fiber network is dispersed. Electrospinning is used for the deposition of the fiber network whereas the continuous epitaxial phase is spin-coated. Good coating is observed between the liquid precursor of the continuous oxide and the fibers and remarkably, epitaxial (001) growth of the $\mathrm{YBa}_{2} \mathrm{Cu}_{3} \mathrm{O}_{7-\mathrm{x}}$ is not affected by the presence of the fiber network because both oxides do not react to each other. Topological continuity of the continuous phase is probed by electrical conductivity measurements, rendering nearly the same values reported for fiber-free films. Mechanical properties are determined by nanoindentation at low penetration depths to avoid the effect of the single crystal beneath the composite. Enhanced mechanical properties are found (hardness, Young's modulus, elastic recovery and wear resistance).

Keywords: Ceramic fiber, electrospinning, thin film, chemical solution deposition, mechanical properties, mechanical testing 


\section{Introduction}

Composite materials take advantage in a synergistic manner of the individual properties of each constituent and the properties arising from the interaction between the two or more components. Both the intrinsic properties of the constituent materials (e.g., composition, grain size, etc.) as well as the morphology (particles, rods, nanowires, multilayers, etc.) play a key role in the resulting composite behavior. Some clear examples could be metal-ceramic composites, where metals contributes to the ductility and ceramics adds hardness, or the widely-used fiber-glass reinforced plastics, where both tension and compression strengths of the plastic matrix are significantly improved as a result of the stiffer glass fibers.

In sol-gel derived films, composite mechanical reinforcement has been shown to lead to crack-free thick films of different oxides by dispersing the already crystallized powders in the metalorganic precursor film. The strong bonding between the soft metalorganic matrix and the oxide particles yields a stiff composite film able to withstand mechanical stresses during shrinkage [1].

Regarding approaches for preparing composites at the nanoscale in sol-gel derived thin films for different purposes, the spontaneous phase separation and self-organization during crystallization has been also used, like in the case of magnetoelectric composite films of ferrimagnetic spinel $\mathrm{CoFe}_{2} \mathrm{O}_{4}$ and piezoelectric perovskite $\mathrm{Pb}(\mathrm{Zr}, \mathrm{Ti}) \mathrm{O}_{3}$ [2] or nanoinclusions of secondary phases of oxides like $\mathrm{Ba}_{2} \mathrm{YTaO}_{6}$ or $\mathrm{BaZrO}_{3}$ in biaxiallytextured $\mathrm{YBa}_{2} \mathrm{Cu}_{3} \mathrm{O}_{7}$ superconductor matrix, where strain at the interface between both phases produces strong flux pinning defects for improved performance [3]. This general strategy also works when one component crystallizes out of a glassy matrix, where simple metal oxides crop out of the $\mathrm{SiO}_{2}$ glassy matrix prepared by polyalkylsiloxanes containing metal halides to yield nanocomposite films useful as optical gas sensors [4]. On the other hand, nanocomposite films in which one of the constituents has 1D geometry (i.e., nanowires) are potentially interesting for exploiting the larger stiffness of the 1D nanobject for mechanical reinforcement, like in the case of much studied carbon nanotubes in bulk, film or fiber polymers $[5,6]$.

In this work, we report the preparation and mechanical characterization of a composite superconductor thin film comprised of a electrospun $\mathrm{BaZrO}_{3}(\mathrm{BZO})$ oxide fiber network embedded in the epitaxial layer of the high temperature superconductor oxide 
$\mathrm{YBa}_{2} \mathrm{Cu}_{3} \mathrm{O}_{7}$ (YBCO). $\mathrm{BaZrO}_{3}$ was selected as the oxide forming the fibers due to its recognized immiscibility with the YBCO, improving flux pinning properties [7]. Special attention was given to the mechanical properties of the composite film at the nanoscale in order to investigate the effect of the fibers, which is relevant in the manipulation of the superconductor tapes during manufacturing, tolerance to mechanical stresses in operation as well as in quench events.

\section{Experimental}

The preparation of the BZO precursor solution follows the procedure described by Calleja et al [8]. Barium acetate (Fluka) and zirconium 2,4-pentadionate (Alfa Aesar) are dissolved in glacial acetic acid (Panreac). Total metal concentration is $0.25 \mathrm{M}$. Afterwards, polyvinylpyrrolidone or PVP with $\mathrm{M}_{\mathrm{w}}=1,3 \cdot 10^{6}$ (Alfa Aesar) is added in $10 \% \mathrm{w} / \mathrm{v}$ concentration in order to increase viscosity to $\sim 600 \mathrm{mPa} \cdot \mathrm{s}$.

Electrospinning was then performed in a home-made apparatus. The applied voltage was set at $10 \mathrm{kV}$ and the distance between tip and collector was $100 \mathrm{~mm}$. A $5 \times 5 \mathrm{~mm}^{2}$ (100)-oriented lanthanum aluminate (LAO) single crystal (Crystec) was placed in the center of the copper collector and on-axis with the syringe tip. In order to have a high density of fibers on the substrate, the deposition time was set to 5 minutes. Once deposited, the fibers were dried at $120^{\circ} \mathrm{C}$ for 10 minutes.

Once deposited, the coated single crystals were subjected to thermal treatment in a tubular resistance furnace at $800^{\circ} \mathrm{C}$ for 2 hours in an oxygen flow of $0.1 \mathrm{l} / \mathrm{min}$. Heating and cooling ramps were $10^{\circ} \mathrm{C} / \mathrm{min}$. After the thermal treatment, films were ultrasonicated to remove fiber networks which are not well adhered.

The YBCO heteroepitaxial oxide was grown by the so-called TFA route [11]. The trifluoroacetates of $\mathrm{Y}, \mathrm{Ba}$ and $\mathrm{Cu}(\mathrm{II})$ cations were prepared following the process of Roma et al. [19], which yields a methanolic solution with a total metal concentration of 1.5 M. Such solution was deposited on the calcined BZO fiber network by spin-coating at $6000 \mathrm{rpm}$ with $2 \mathrm{~s}$ of acceleration. Subsequently, the YBCO was pyrolyzed at $3^{\circ} \mathrm{C} / \mathrm{min}$ up to $210^{\circ} \mathrm{C}$ and then $1^{\circ} \mathrm{C} / \mathrm{min}$ up to $310^{\circ} \mathrm{C}$ for 30 minuts in oxygen flow of $0.12 \mathrm{l} / \mathrm{min}$. After this, the $\mathrm{YBCO}$ was grown at $810^{\circ} \mathrm{C}$ for 3 hours.

In order to identify the effect of BZO fibers on the oxide matrix, a fiber-free sample was grown on LAO single crystal with same processing conditions using the same solution batch. 
The mechanical properties (hardness and reduced elastic modulus) were evaluated by nanoindentation, operating in the load control mode, using an UMIS setup from Fischer-Cripps Laboratories equipped with a Berkovich pyramidal-shaped diamond tip. The value of maximum applied force was chosen to be $0.5 \mathrm{mN}$ to ensure that the maximum penetration depth during the tests was kept below one tenth of the overall film thickness. This is a necessary condition to avoid any possible influence from the substrate on the resulting mechanical properties of the film [20]. The thermal drift during nanoindentation was kept below $0.05 \mathrm{~nm} / \mathrm{s}$. Proper corrections for the contact area (previously calibrated using a fused quartz specimen), instrument compliance, and initial penetration depth were applied. The hardness $(\mathrm{H})$ and reduced elastic modulus $\left(\mathrm{E}_{\mathrm{r}}\right)$ values were calculated from the load-displacement curves at the beginning of the unloading segment using the method of Oliver and Pharr [21]. From the initial unloading slope, the contact stiffness, $\mathrm{S}$, can be determined as:

$$
S=\frac{d P}{d h}
$$

where $\mathrm{P}$ and $\mathrm{h}$ denote, respectively, the applied force and the penetration depth during nanoindentation. The elastic modulus is evaluated based on its relationship with the contact area, A, and contact stiffness:

$$
S=\beta \frac{2}{\sqrt{\pi}} E_{r} \sqrt{A}
$$

Here, $\beta$ is the King's factor, which depends on the geometry of the indenter $(\beta=1.034$ for a Berkovich indenter) [20], and $\mathrm{E}_{\mathrm{r}}$ is the so-called reduced Young's modulus, which takes into account the elastic displacements that occur in the specimen (with Young's modulus $E$ and Poisson's ratio $v$ ), as well as in the diamond indenter (with elastic constants $E_{i}=1140 \mathrm{GPa}$ and $\left.v_{i}=0.07\right)$ :

$$
\frac{1}{E_{r}}=\frac{1-v^{2}}{E}+\frac{1-v_{i}^{2}}{E_{i}}
$$

Hardness is calculated from the following expression:

$$
H=\frac{P_{\operatorname{Max}}}{A}
$$


where $\mathrm{P}_{\mathrm{Max}}$ is the maximum load applied during nanoindentation. Finally, the elastic recovery can be evaluated as the ratio between the elastic and the total (plastic + elastic) energies during nanoindentation, $\mathrm{W}_{\mathrm{el}} / \mathrm{W}_{\text {tot }}$. These energies can be calculated from the nanoindentation experiments as the areas between the unloading curve and the $\mathrm{x}$-axis $\left(\mathrm{W}_{\mathrm{el}}\right)$ and between the loading curve and $\mathrm{x}$-axis $\left(\mathrm{W}_{\text {tot }}\right)[22]$. The results presented here represent the statistical average of a set of 200 indentations for each sample.

Resistivity of the grown composite film at ambient temperature was made in a PPMS with a Van der Pauw configuration using $5 \mu \mathrm{A}$. The electrodes were painted with silver paste at the four corners of the squared film.

A General Area Detector Diffraction System (GADDS) from Bruker with twodimensional detector was used for the characterization by X-ray diffraction. Further information about YBCO surface, porosity and nucleation assessment was obtained using a FEI Quanta 200 FEG. DC magnetic measurements were conducted with a Superconducting Quantum Interference Device magnetometer provided with a $9 \mathrm{~T}$ superconducting coil, which allowed a temperature variation in the sample space from 5 $\mathrm{K}$ to room temperature. Samples were mounted with the dc magnetic field applied perpendicular to the substrate and standard magnetic hysteresis loops were measured, cooling the sample in zero field (ZFC).

\section{Results and discussion}

In Fig. 1, a sketch of the whole process is depicted. The sequence starts with the electrospinning of the BZO precursor solution on a (001)-oriented lanthanum aluminate (LAO) single crystal, followed by heat treatment at $800^{\circ} \mathrm{C}$. In-plane randomly-oriented network of fibers is present at this stage, with fiber diameter ranging from ca. 200-500 $\mathrm{nm}$ and length of tens or hundreds of $\mu \mathrm{m}$. Continuous fibers can be found in direct contact with the substrate whereas others cross above the former, being partially broken during the thermal decomposition. The tension forces in the network during the shrinkage likely lead to fragmentation of these fibers. On the other hand, the fibers adhered to the substrate maintain the continuous filament structure. At this point, the 
fibers are made of nanocrystalline $\mathrm{BaZrO}_{3}$ since organics have been removed during the heating process [8].

In a third step, YBCO precursor solution is spin-coated on the single crystal carrying the BZO fiber network and thermally decomposed to remove organics. After that, the nanocrystalline precursor of $\mathrm{YBCO}$ is optically transparent and allows the observation of the fiber network (Fig. 2). Most of the fibers are embedded in the precursor matrix while some pieces protrude outwards. Good coating of the YBCO solution is thus concluded. Remarkably, no cracks appeared even at the corners and edges where thickness is known to be always higher and more prone to cracking.

Finally, the YBCO film is crystallized at $810^{\circ} \mathrm{C}$. Once grown, the composite film darkens to a black colour and loses the transparency. Thickness is estimated to be 250 $\mathrm{nm}$.

The morphology of the composite film by field-emission scanning electron microscopy (FESEM) is displayed in Fig. 3a and 3b. In Figure 3a, some fibers are identified which are at a higher altitude than the YBCO matrix. A zoomed image (inset Fig. 3a) shows that YBCO has a terrace-like morphology typical of c-axis orientation resuting from the heteroepitaxial growth from the LAO (100) substrate. By looking at the backscattered FESEM image in Fig. 3b, fibers inside the YBCO are identified owing to the increased sensitiveness of backscattered electrons to the film depth. The BZO content can be estimated taking the whole length of the observed fibers in the image and considering an average diameter of $500 \mathrm{~nm}$. This gives a volume fraction of $3.7 \%$ of BZO fibers inside the matrix although this is somewhat overestimated since fibers are not so thick in average, as discussed previously. In addition, it is interesting to note that the surface of YBCO appears substantially free of any segregated particles, in contrast to BZO-free films prepared with the same solution and thermal treatments (Fig. 4) . This is still not properly understood and calls for further study of the origin and evolution of typical copper-rich segregation and how it is changed by the BZO fibers in our case.

$\chi$-2 $2 \theta$ bidimensional X-ray pattern in Fig. 5 is used to assess the crystalline orientation of the composite film. Regarding the YBCO, only (001) reflections of the ternary oxide are detected, indicating full c-axis epitaxial growth on the (001)-oriented LAO single crystal. In addition, the BZO crystals forming the fiber network are mostly randomlyoriented . This is supported by the BZO (110) polycrystalline ring observed at ca. $2 \theta \approx$ 
$30^{\circ}$. Furthermore, the (001) and (002) reflections of the BZO also appears as a spot, which would account for the small epitaxial fraction of BZO particles coming from the adhered BZO fibers grown cube-on-cube with the LAO substrate [9].

At this point, the c-axis orientation of the fiber-containing YBCO matrix oxide should be highlighted since the existing BZO fiber network does not seem to hinder the correct epitaxial growth. This also means that YBCO does not nucleate on the BZO fibers during the growth process, in which case some polycrystalline YBCO fraction would have been identified by X-ray diffraction. This result is in accordance with that of Gutierrez et al. [7] where randomly-oriented BZO isolated nanoparticles grown by insitu chemical approaches were found surrounded by the c-axis oriented YBCO grains provided that BZO nucleates and grow before YBCO onset crystallization [10].

An interesting question now is to what extent the electrically insulating BZO fibers block the normal state circulation of electrons in the YBCO metallic matrix. To answer this, resistivity measurements were undertaken at ambient temperature for the YBCO/BZO composite, showing a resistivity value of $0.59 \mathrm{mOhm} \cdot \mathrm{cm}$ at $293 \mathrm{~K}$ assuming $250 \mathrm{~nm}$ for the YBCO thickness. Assuming that current flows mostly along the ab-plane, the obtained resistivity compares well with ab-plane resistivity obtained in high $\mathrm{J}_{\mathrm{c}}$ pure YBCO-TFA layers of similar thickness, where values between 0.3 and 0.7 $\mathrm{mOhm} \cdot \mathrm{cm}$ were found [11]. This is a strong indication that current can still easily percolate through the ab-plane of the YBCO epitaxial layer around the BZO fiber network without being blocked by them. At this respect, the embedded BZO fibers which form bridges between underlying fibers during the electrospinning step neither seem to significantly block the current, providing more conduction paths in the YBCO layer. This would also imply that indeed YBCO precursor solution is able to percolate and fill the voids in the fiber network during spin-coating, leading to nearly unperturbed resistivity characteristics.

Superconducting properties of this composite film have been also studied. Assuming $250 \mathrm{~nm}$ as the film thickness, critical current density $J_{c}$ was 0.7 and $0.1 \mathrm{MA} / \mathrm{cm}^{2}$ at 5 and $77 \mathrm{~K}$, respectively. In Fig. 6, zero-field cooled DC-susceptibility curve is shown. A $\mathrm{T}_{\mathrm{c} \text {,onset }}$ of $89 \mathrm{~K}$ is obtained together with a broad superconducting transition, which is attributed to the granularity of the YBCO regions inside the BZO fibers. In contrast to the the resistivity at ambient temperature, superconducting properties are clearly 
reduced with the presence of BZO fibers. In this respect, the fibers allow the penetration of magnetic flux and cause the material to become granular.

The fact that the c-axis orientation of the YBCO layer is preserved despite the presence of the BZO fiber network is a good opportunity to test the mechanical properties of the composite film and compare them with the pristine YBCO sample. At this respect, BZO is harder and stiffer than YBCO if reported values of Vickers hardness and Young's modulus in polycrystalline bulks are considered [12,13]. This was further confirmed by enhanced mechanical properties in polycrystalline YBCO samples with $\mathrm{Zr}$ additions leading to BZO [14]. Representative load-displacement nanoindentation curves are shown in Fig. 7. The maximum penetration depth achieved at the end of the loading is smaller for the YBCO film containing the fibers, suggesting that the fibers play a reinforcing role, increasing the hardness of the composite YBCO film. Furthermore, the slope of the unloading segment (which is denoted as contact stiffness, see Equation 1) is also steeper in the film with fibers. This brings about an increase of $E_{r}$, as shown in Table 1, where the mechanical properties of pristine and composite YBCO films are shown. Besides the direct role of the fibers themselves, indirect effects arising from the presence of the fibers (i.e., changes in surface roughness, lack of precipitated particles, residual stresses, etc.) may also contribute to the observed enhanced mechanical properties.

From a tribological point of view, the wear resistance of a thin film not only depends on its hardness (typically harder materials exhibit a higher wear resistance) but also on the reciprocal of the Young's modulus. The elastic strain to failure, which is related to the ratio $\mathrm{H} / \mathrm{E}_{\mathrm{r}}$, has been claimed to be a suitable parameter to indirectly assess the wear resistance of a coating, even more than $\mathrm{H}$ itself $[15,16]$. The presence of the $\mathrm{BaZrO}_{3}$ fibers increases the wear resistance, thus making the superconducing film more robust in applications requiring some mechanical friction. Finally, the elastic recovery, $\mathrm{W}_{\mathrm{el}} / \mathrm{W}_{\text {tot }}$, also increases with the presence of the fibers in the superconducing films. This parameter is of particular interest in applications where the film might have to resist against impact loading. In such a case, the elastic recovery indicates how much energy is released from the material after being loaded. It has been previously reported that the elastic recovery is directly proportional to the $\mathrm{H} / \mathrm{E}_{\mathrm{r}}$ ratio $[17,18]$. The results presented here are thus in agreement with this previously established correlation. 



\section{Conclusions}

In summary, a new method for producing composite oxide thin films with enhanced mechanical properties is presented. This method consists of: electrospinning precursor oxide fibers on top of crystalline templates, crystallizing such fibers to obtain a fiber network and epitaxially growing a second oxide phase by chemical solution deposition on the fiber network. Using this procedure, films made of BZO electrospun fibers embedded inside a c-axis oriented YBCO matrix are obtained. Immiscibility of both oxides -fiber and matrix- seems mandatory to avoid chemical reactions and keep the composite film in the desired structure. Mechanical reinforcement of the fiber network was proven by nanoindentation. The enhanced mechanical performance of the composites might not arise solely from the presence of the fibers but also from changes in the matrix occurring during the synthesis process (i.e., during the chemical decomposition of the organics). Apart from the mechanical reinforcement, other interesting composite films based on this preparation scheme can be designed and prepared by smartly choosing the immiscible oxides and their functionality in order to exploit their synergistic effect in real devices.

\section{Acknowledgements}

This work was supported by MINECO (MAT2011-28874-C02, MAT2011-27380-C0201, CSD2007-0041, IPT-2011-1090-920000, Ramón y Cajal program on behalf of AC); Generalitat de Catalunya (Pla de Recerca SGR-770, 2014-SGR-1015 and XaRMAE).

\section{References}

1. Barrow DA, Petroff TE, Sayer M (1995) Surf Coat Technol 76-77:113-118.

2. Wan JG, Wang XW, Wu YJ, Zeng M, Wang Y, Jiang H, Zhou WQ, Wang GH, Liu J-M (2005) Appl Phys Lett 86:122501. 
3. Coll M, Ye S, Rouco V, Palau A, Guzman R, Gazquez J, Arbiol J, Suo H, Puig T, Obradors X (2013) Supercond Sci Technol 26:015001 .

4. Martucci A, Bassiri N, Gugliemi M, Armelao L, Gross S, Pivin JC (2003) J Sol-Gel Sci Technol 26:993-996.

5. Coleman JN, Khan U, Gun'ko YK (2006) Adv Mat 18:689-706.

6. Cadek M, Coleman JN, Barron V, Hedicke K, Blau WJ (2002) Appl Phys Lett $81: 5123-5125$.

7. Gutierrez J, Llordes A, Gazquez J, Gibert M, Roma N, Ricart S, Pomar A, Sandiumenge F, Mestres N, Puig T, Obradors X (2007) Nat Mater 6:367-373.

8. Calleja A, Ricart S, Granados X, Palmer X, Solano E, Cano F, Tornero J A, Puig T, Obradors X (2012) CrystEngComm 14: 4686-4691.

9. Abellán P, Sandiumenge F, Casanove M-J, Gibert M, Palau A, Puig T and Obradors X (2011) Acta Mater 59: 2075-2082.

10. Miura M, Yoshizumi M, Izumi T, Shiohara Y (2010) Supercond Sci Technol 23: 014013.

11. Obradors X, Puig T, Pomar A, Sandiumenge F, Piñol S, Mestres N, Castaño O, Coll M, Cavallaro A, Palau A, Gazquez J, Gonzalez JC, Gutierrez J, Roma N, Ricart S, Moreto JM, Rossell MD, van Tendeloo G (2004) Supercond Sci Technol 17: 10551064

12. Vassen R, Cao X, Tietz F, Basu D, Stöver D (2000) J Am Ceram Soc 83: 2023 2028. 
13. Kölemen U, Çelebi S, Karal H, Öztürk A, Çevik U, Nezir S, Görür O (2004) Phys. Status Solidi 241:274-283.

14. Oka T, Ogasawara F, Itoh Y, Suganuma M, Mizutani U (1990) Jpn J Appl Phys 29: 1924-1933.

15. Pellicer E, Varea A, Pane S, Nelson BJ, Menendez E, Estrader M, Suriñach S, Baro MD, Nogues J, Sort J. (2010) Adv Funct Mater 20: 983-991.

16. Leyland A, Matthews A (2000) Wear 246: 1-11.

17. Pellicer E, Pane S, Sivaraman KM, Ergeneman O, Suriñach S, Baro MD, Nelson BJ, Sort J. (2011) Mater Chem Phys 130: 1380-1386.

18. Cheng YT, Cheng CM (1998) Appl Phys Lett; 73: 614-616.

19. Roma N, Morlens S, Ricart S, Zalamova K, Moreto JM, Pomar A, Puig T, Obradors X (2006) Supercond Sci Technol 19: 521-527.

20. Fischer-Cripps AC Nanoindentation. Springer, 2004.

21. Oliver WC, Pharr GM (1992) J Mater Res 7:1564-1583.

22. Fornell J, Gonzalez S, Rossinyol E, Suriñach S, Baro MD, Louzguine-Luzgin DV, Perepezko JH, Sort J, Inoue A (2010) Acta Mater 58: 6256-6266. 


\section{$\underline{\text { Figures }}$}

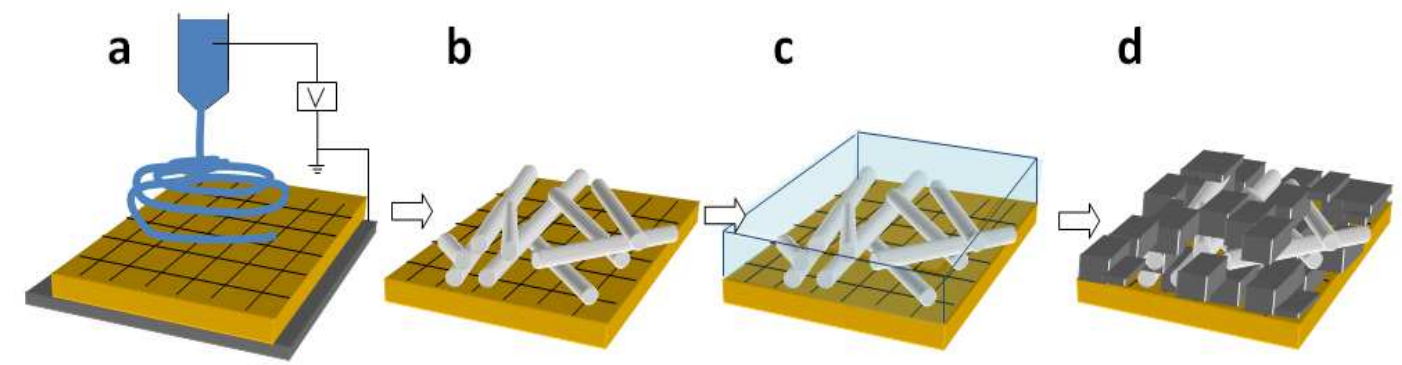

Figure 1. a) Electrospinning of BZO precursor fibers on LAO single crystal. b) BZO fiber network after thermal treatment. c) Spin-coating of metalorganic YBCO precursor solution on top of the BZO fiber network. d) Epitaxial film with embedded BZO fibers after the crystal growth of YBCO. 


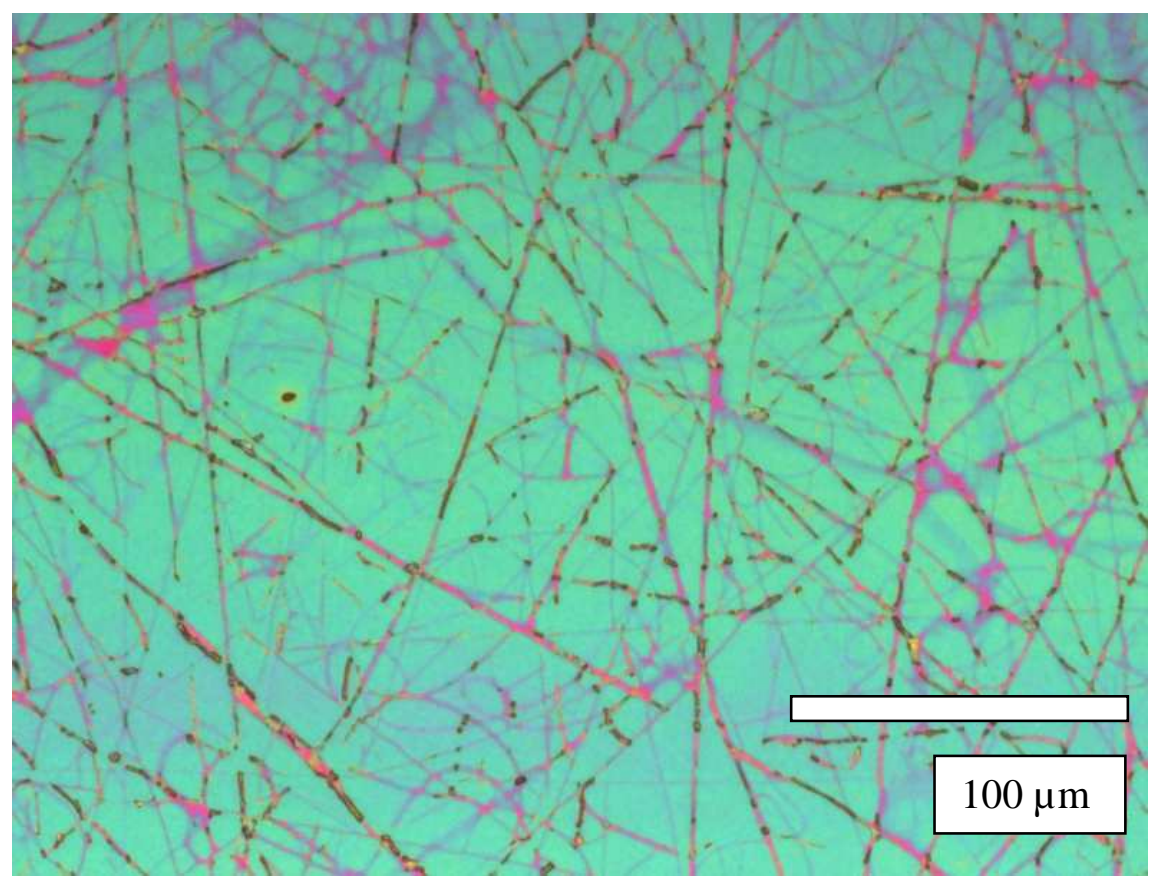

Figure 2. Optical image corresponding to the pyrolyzed YBCO-TFA layer with the BZO fiber network, which can be clearly observed. 
(a)

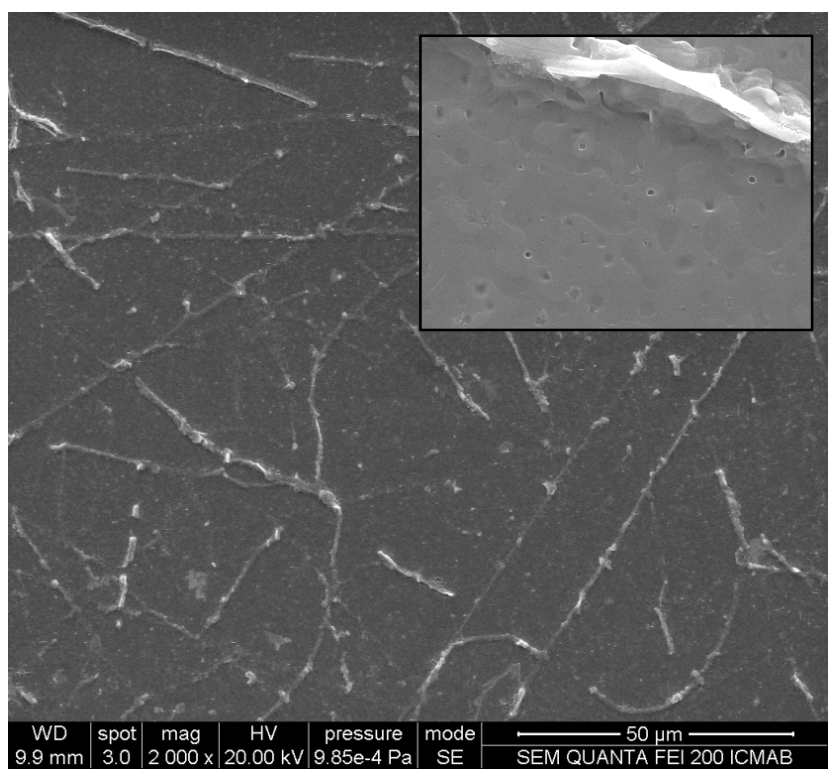

(b)

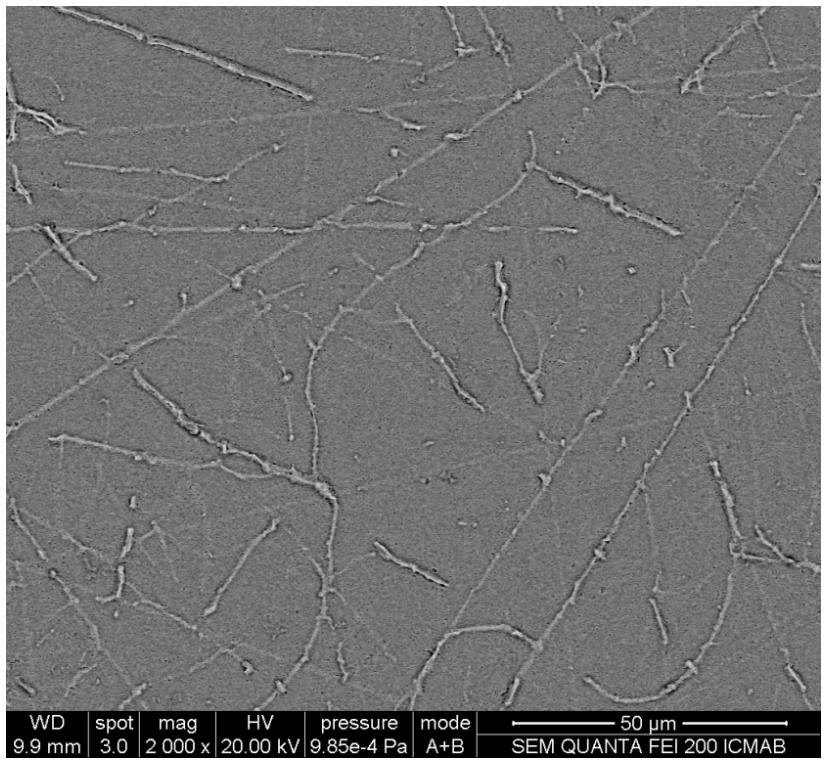

Figure. 3. a) Secondary electron FESEM image of the obtained nanocomposite. The BZO fiber network in the YBCO layer can still be observed after the YBCO conversion at high temperature. Inset showing a close-up secondary electron FESEM image showing high density c-axis oriented YBCO region near a BZO fiber. b) Backscattered FESEM image that reveals embedded BZO fibers inside the YBCO epitaxial matrix. 


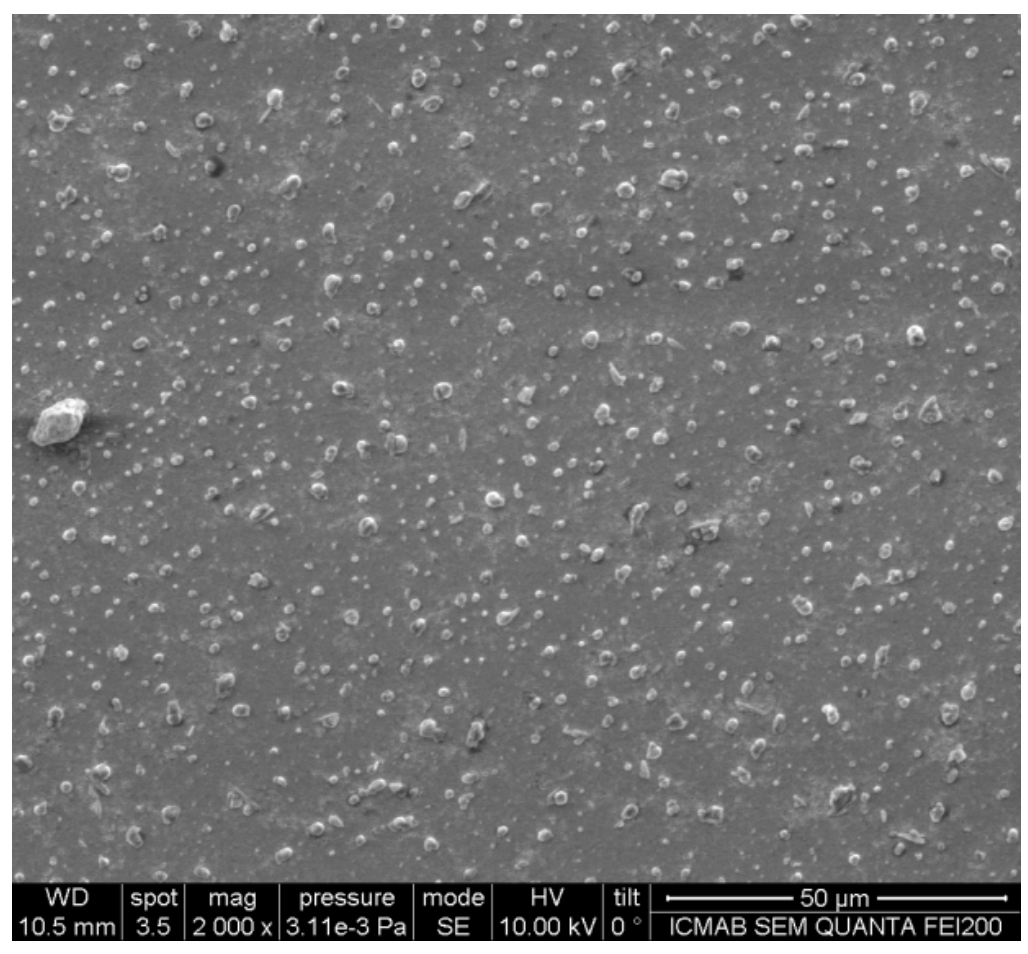

Figure 4. FESEM image corresponding to the BZO-free YBCO film prepared for comparison purposes. 

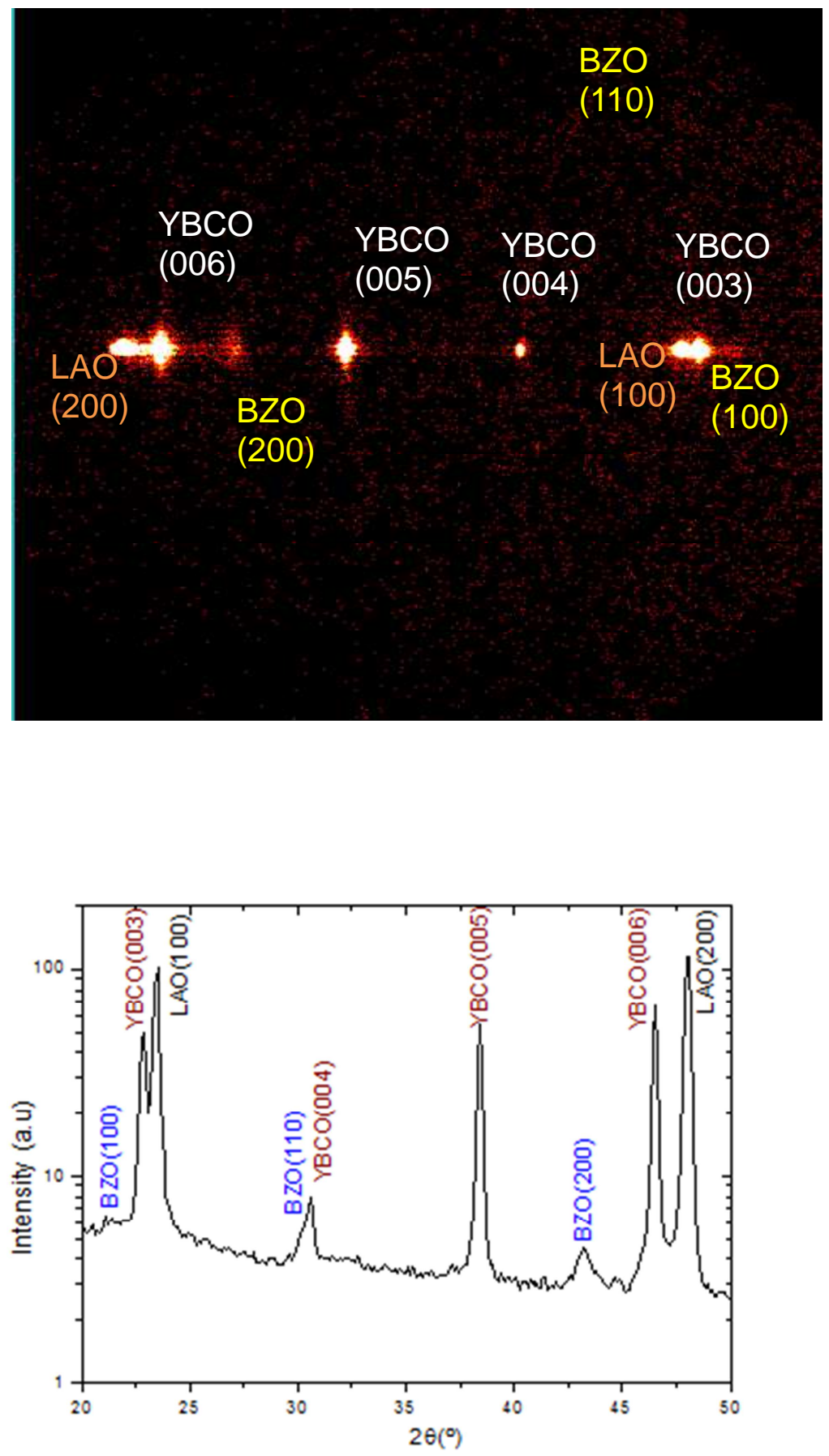

Figure 5. a) GADDS frame of the $\mathrm{YBCO} / \mathrm{BZO}$ fiber and b) integrated $\theta-2 \theta$ diffraction pattern. 


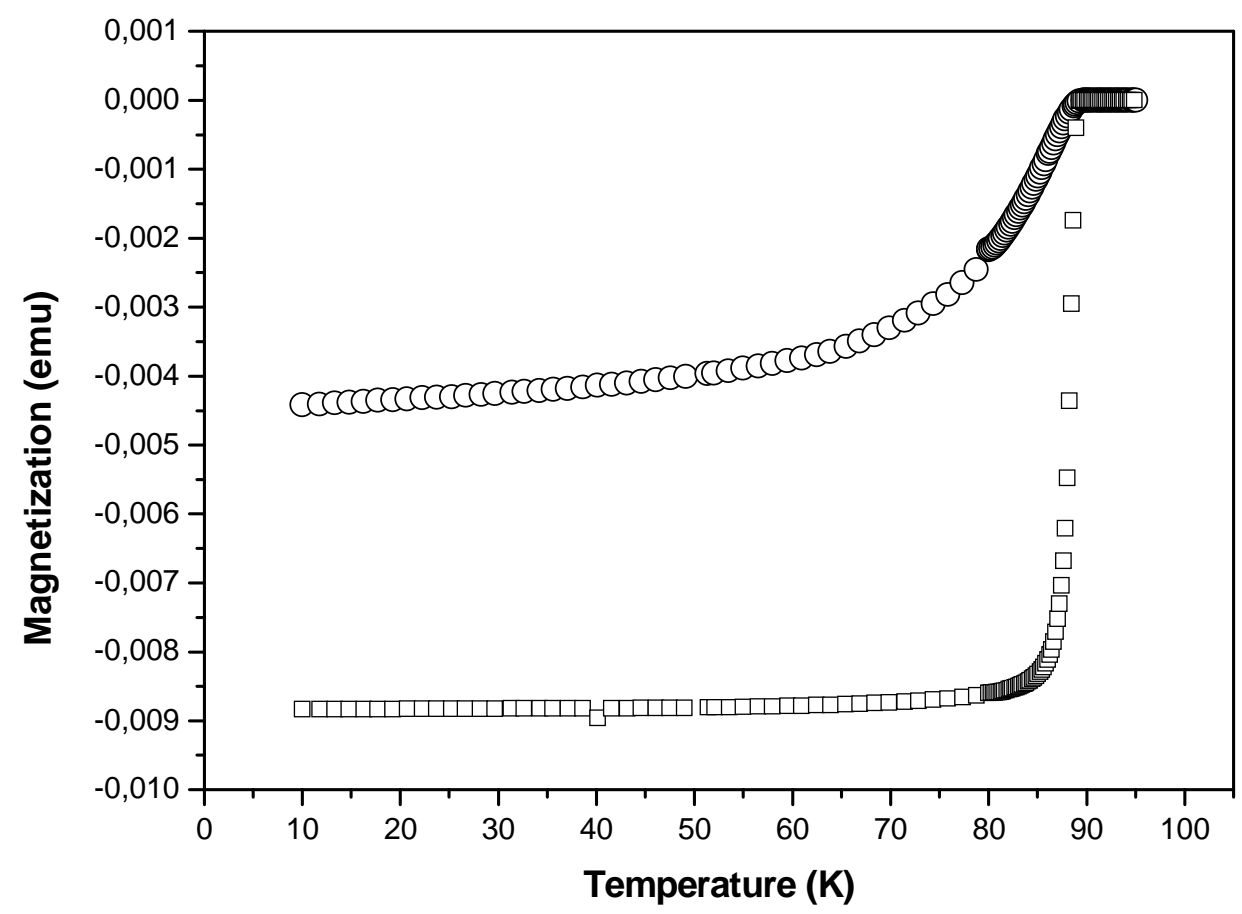

Figure 6. Zero-field cooled DC-susceptibility as a function of temperature, showing a $\mathrm{T}_{\mathrm{c}, \text { onset }}=89 \mathrm{~K}$ for the $\mathrm{YBCO} / \mathrm{BZO}$ composite film $(\mathrm{O})$ and $\mathrm{BZO}$-free $\mathrm{YBCO}$ film shown for comparison $(\square)$. $\mathrm{H}=2$ Oe. 


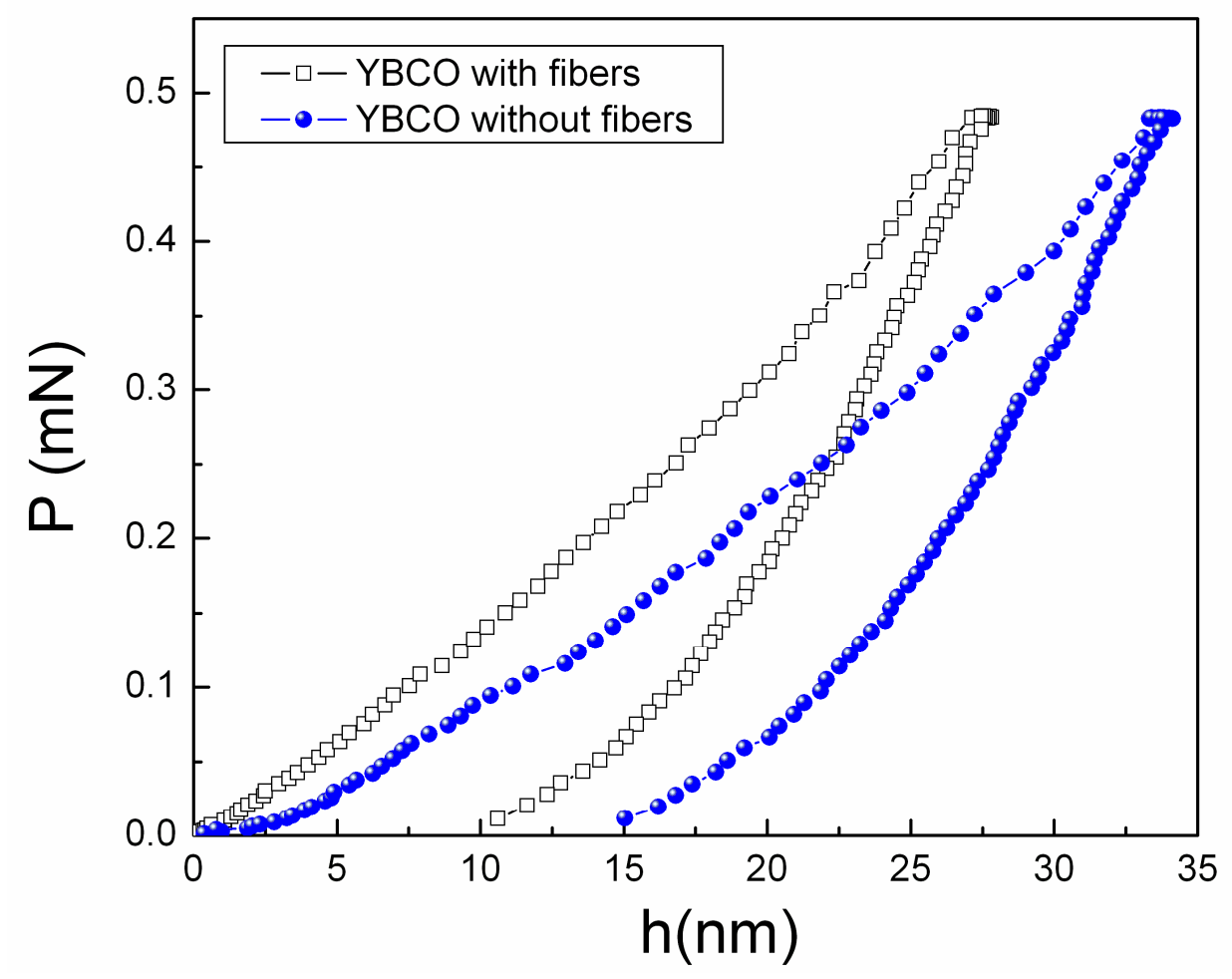

Figure 7. Representative load-displacement nanoindentation curves corresponding to the YBCO films with and without the presence of electrospun fibers. 
Table 1. Summary of the mechanical properties obtained by nanoindentation.

\begin{tabular}{|cccccc|}
\hline Sample & $\begin{array}{c}\mathrm{H} \\
(\mathrm{GPa})\end{array}$ & $\begin{array}{c}\mathrm{E} \\
(\mathrm{GPa})\end{array}$ & $\mathrm{W}_{\mathrm{el}} / \mathrm{W}_{\text {tot }}$ & $\mathrm{W}_{\mathrm{pl}} / \mathrm{W}_{\text {tot }}$ & $\mathrm{H} / \mathrm{E}_{\mathrm{r}}$ \\
\hline YBCO & 9.3 & 176.55 & 0.4910 & 0.5090 & 0.0529 \\
\hline $\begin{array}{c}\text { YBCO+BZO } \\
\text { composite }\end{array}$ & 11.8 & 211.34 & 0.5255 & 0.4744 & 0.0558 \\
\hline
\end{tabular}

